\title{
Téoros
}

Revue de recherche en tourisme

TEE̊ROS

\section{Le ciné-tourisme comme pratique allographique}

\section{Hécate Vergopoulos et Michaël Bourgatte}

Volume 30, numéro 1, 2011

Ciné-tourisme

URI : https://id.erudit.org/iderudit/1012113ar

DOI : https://doi.org/10.7202/1012113ar

Aller au sommaire du numéro

\section{Éditeur(s)}

Université du Québec à Montréal

\section{ISSN}

0712-8657 (imprimé)

1923-2705 (numérique)

Découvrir la revue

\section{Citer cet article}

Vergopoulos, H. \& Bourgatte, M. (2011). Le ciné-tourisme comme pratique allographique. Téoros, 30(1), 99-107. https://doi.org/10.7202/1012113ar

\section{Résumé de l'article}

En partant du constat que la nature des destinations et des expériences touristiques se diversifie, se multiplie et se renouvelle sans cesse (au point que des lieux touristiques en deviennent carrément loufoques et même follement inatteignables), nous avons cherché à savoir de quelle manière le cinéma participe à la qualification ou requalification de certains lieux apparemment banals pour en faire des destinations dignes d'un intérêt touristique.

Cet article montre que les dispositifs de médiation touristique n'hésitent pas à convoquer des films pour transformer des fragments d'espace en sites touristiques, des lieux apparemment anodins en choses à voir. Il met ensuite en évidence la manière dont certains lieux tirent parti de leur inscription dans un récit filmique pour s'assurer une existence touristique par la mise en exposition de traces cinématographiques. Il dévoile enfin que le ciné-tourisme repose sur une écriture de l'autre qui garantit une carrière ciné-touristique à certains lieux mis en scène dans des films.

Cette inscription de la destination touristique dans le film, le maintien de traces dans des lieux ayant servi à des tournages ainsi que les opérations de qualifications de fragments d'espace au titre de sites ciné-touristiques forment ce que nous appelons « une boucle alllographique ». Cette dernière peut être saisie comme une compétence communicationnelle des acteurs du ciné-tourisme : pour mettre les lieux en culture, et plus particulièrement en tourisme, ils doivent savoir composer avec une intertextualité qui se joue entre espaces, objets et discours.
Ce document est protégé par la loi sur le droit d'auteur. L'utilisation des services d'Érudit (y compris la reproduction) est assujettie à sa politique d'utilisation que vous pouvez consulter en ligne.

https://apropos.erudit.org/fr/usagers/politique-dutilisation/ 


\title{
Le ciné-tourisme comme pratique allographique
}

\author{
Hécate VERGOPOULOS \\ Docteure en sciences de l'information et de la communication \\ Ph.D. en muséologie \\ Chercheuse au GRIPIC (Celsa-Université Paris IV) \\ hecate.vergopoulos@gmail.com

\section{Michaël BOURGATTE} \\ Docteur en sciences de l'information et de la communication \\ Postdoctorant et chercheur à Télécom ParisTech \\ Département SES, UMR CNRS LCTI \\ michael.bourgatte@gmail.com
}

\begin{abstract}
RÉSUMÉ: En partant du constat que la nature des destinations et des expériences touristiques se diversifie, se multiplie et se renouvelle sans cesse (au point que des lieux touristiques en deviennent carrément loufoques et même follement inatteignables), nous avons cherché à savoir de quelle manière le cinéma participe à la qualification ou requalification de certains lieux apparemment banals pour en faire des destinations dignes d'un intérêt touristique.

Cet article montre que les dispositifs de médiation touristique n'hésitent pas à convoquer des films pour transformer des fragments d'espace en sites touristiques, des lieux apparemment anodins en choses à voir. II met ensuite en évidence la manière dont certains lieux tirent parti de leur inscription dans un récit filmique pour s'assurer une existence touristique par la mise en exposition de traces cinématographiques. II dévoile enfin que le ciné-tourisme repose sur une écriture de l'autre qui garantit une carrière ciné-touristique à certains lieux mis en scène dans des films.

Cette inscription de la destination touristique dans le film, le maintien de traces dans des lieux ayant servi à des tournages ainsi que les opérations de qualifications de fragments d'espace au titre de sites ciné-touristiques forment ce que nous appelons «une boucle alllographique». Cette dernière peut être saisie comme une compétence communicationnelle des acteurs du ciné-tourisme : pour mettre les lieux en culture, et plus particulièrement en tourisme, ils doivent savoir composer avec une intertextualité qui se joue entre espaces, objets et discours.
\end{abstract}

Mots-clés: Allographie, ciné-tourisme, marqueur, stéréotype, trace.

On considère volontiers qu'on a conquis, en termes de tourisme, tous les espaces du monde et qu'il n'y a pas un sentier qu'un touriste n'ait déjà parcouru. En conséquence, les industries touristiques s'engagent dans la conquête improbable de territoires imaginaires (Peeters et Schuiten, 2002; Richard, 2006) ou de territoires a-touristiques (Cohen, 2006). Plus encore, elles cherchent à mettre en tourisme des espaces ordinaires, des lieux de la quotidienneté qui ont su trouver une existence autre à travers des ouvres de fiction (Hyman et Rea, 2010 : 30-47; Casazza, 2003). Le ciné-tourisme participe à l'ouverture de cette terra incognita.

Dans cet article, il sera question d'espaces de pratiques ordinaires requalifiés en lieux touristiques extraordinaires, d'œuvres cinématographiques qui travaillent à la construction de destinations touristiques ou encore de dispositifs de médiation qui n'hésitent pas à mettre en relation des univers narratifs et des espaces topographiques. La thèse que cet article cherche à défendre est qu'il existe une pratique d'écriture ciné-touristique engendrée à la fois par les lieux, les films qui les convoquent et les dispositifs de médiation qui les mettent en relation. Cette pratique assure aux lieux une carrière touristique.

À partir d'une approche communicationnelle d'un corpus de lieux mis en scène dans des films ou médiatisés en tant que tels par des acteurs touristiques, nous avons cherché à comprendre comment le rapport entre le film et le lieu permet de construire un espace de pratiques touristiques. Nous nous sommes intéressés à trois types de médiation des lieux ciné-touristiques que nous avons sélectionnés pour leur complémentarité. À travers ces médiations, nous avons souhaité questionner l'existence d'un phénomène de circulation de valeurs et de représentations associé aux lieux ciné-touristiques.

La première médiation est celle que réalisent les acteurs de l'édition touristique (nous avons ici focalisé notre attention 
sur le Katz's Deli, restaurant utilisé dans le film When Harry Met Sally, tourné en 1989 et dirigé par Rob Reiner, dans lequel le personnage de Sally simule son célèbre orgasme devant Harry et qui est presque systématiquement cité dans les guides de voyage sur New York). La deuxième médiation renvoie à la façon dont les acteurs locaux mobilisent le film pour fonder leur identité touristique (nous avons, cette fois, choisi Kalokairi, l'île du film Mamma Mia!, tourné en 2008 par Phyllida Lloyd et le café des 2 Moulins, où travaille Amélie Poulain, l'héroïne éponyme du film de Jean-Pierre Jeunet sorti sur les écrans en 2001). La troisième médiation est celle des acteurs institutionnels qui font du film un outil stratégique de la promotion territoriale (en l'occurrence, la mairie de Paris et ses Parcours cinéma autour du film collectif Paris, je t'aime, réalisé en 2006).

Pour tous ces lieux, nous avons mené une étude des modalités de convocation des films par les différents dispositifs touristiques. De même, nous avons cherché à identifier la façon dont ces lieux intégrés aux circuits ciné-touristiques sont mis en scène dans des films, notamment pour mettre au jour la construction de stéréotypes liés à la destination. Par ailleurs, nous avons jugé nécessaire d'associer à cette approche un travail d'observation situé pour analyser le type de rapport que les touristes entretiennent, d'une part, avec le lieu et, d'autre part, avec le film, en focalisant notre attention sur le cas du café des 2 Moulins. De manière générale, cette analyse communicationnelle cherche à mettre en évidence «le fait que la signification n'est pas seulement du côté des acteurs ou de la situation, mais dans la relation qui existe entre ceux-ci et les objets concrets technosémiotiques qui servent d'opérateurs de la communication» (Davallon et Jeanneret, $2006: 210$ ).

Dans un premier temps, nous verrons comment les dispositifs de médiation mobilisent le film en tant qu'opérateur de transformation d'un fragment d'espace en site touristique. Nous nous attacherons, dans un second temps, à mettre en évidence la façon dont les lieux tirent parti de leur mise en scène filmique pour s'assurer une existence touristique. Enfin, dans un troisième et dernier temps, nous mettrons en relation les dispositifs de médiation touristique, les lieux définis comme sites ainsi que les films en exploitant la notion d' «allographie touristique». Nous montrerons ainsi que le ciné-tourisme repose sur une écriture de l'autre qui garantit une carrière ciné-touristique à certains lieux mis en scène dans des films.

\section{Le film comme opérateur de transformation d'un fragment d'espace urbain en site touristique}

Cette première partie a pour objectif de mettre en évidence la manière dont un lieu qui a fait l'objet d'une mise en scène cinématographique se laisse saisir par les dispositifs de médiation touristique comme site ou chose à voir. Elle tente, plus précisément, de mesurer le rôle assigné au film dans ce processus de requalification.

\section{Définir la chose à voir}

Lorsque Jules Gritti (1967) analyse «les contenus culturels du Guide bleu ", il met en évidence deux stratégies poétiques élaborées par le guide : celle du devoir-circuler et celle du devoirregarder. Le devoir-circuler se définit comme un impératif du parcours. Pour se rendre d'un point à un autre, le touriste doit nécessairement se déplacer. Ce déplacement est moins l'occasion d'une flânerie qu'une contrainte. Le devoir-regarder, quant à lui, renvoie à la confrontation du touriste au site à visiter. Une fois son trajet parcouru, il se doit de passer de la logique de circulation à celle de la station et de la contemplation. En somme, l'espace tel qu'il est défini du point de vue des guides touristiques s'impose comme un composite de deux ensembles : des lieux, pour ainsi dire neutres, qu'il faut traverser sans s'y arrêter et d'autres lieux qui, au contraire, exigent que l'on s'y attarde, qu'on les observe longuement dans le cadre d'une pratique culturelle.

Autrement dit, les guides ne pratiquent pas une écriture de l'«asyndète» au sens où ils ne procèdent pas, à proprement parler, à «l'ellipse des lieux conjonctifs» dont parle Michel de Certeau (de Certeau et al., 1980, r. 1990 : 152-153). En effet, il ne s'agit pas, pour les guides, d'omettre de parler des lieux de circulation. Au contraire, ils les convoquent pour mieux les faire basculer dans un registre de l'utilité qui s'opposerait à celui de la culture représenté par les sites à visiter. Cette dialectique de la circulation et de la station est à la source de la notion de chose à voir. C'est parce qu'il existe des lieux neutres à traverser que ceux qui méritent le détour sont proprement culturels. En somme, le devoir-regarder n'a de sens que dans un rapport complémentaire au devoir-circuler.

Ce que Jules Gritti (1967) met en évidence à propos du Guide bleu est non seulement vrai pour les guides en général, mais aussi pour tous les types de dispositifs de médiation touristique à caractère documentaire qui « disent de [faire] et comment faire» (Adam, 2001). En effet, ce type de textes, qu'il s'agisse de guides imprimés, de documents téléchargeables sur l'Internet ou encore de plaquettes institutionnelles, élaborent une rhétorique injonctive qui a pour objectif d'orienter la pratique touristique en organisant la rencontre du touriste et des choses à voir. C'est notamment le cas des Parcours cinéma proposés par la mairie de Paris.

\section{De l'espace urbain ordinaire au site touristique extraordinaire}

En janvier 2002, le maire de la ville de Paris, Bertrand Delanoë, a créé une Mission cinéma dont l'objectif est de préserver et de promouvoir les richesses cinématographiques de la capitale. À partir de 2006, avec la sortie du film collectif Paris, je t'aime, une série de parcours ciné-touristiques a vu le jour. Ils «invitent les parisiens et les touristes français et étrangers à découvrir Paris, ses quartiers célèbres ou insolites à travers des films réalisés dans la capitale» (mairie de Paris, 2010) Parmi les parcours proposés, on retrouve pêle-mêle les lieux de tournage du Petit Nicolas (Tirard, 2009), de Rush Hour 3 (Ratner, 2007) ou encore de The Devil Wears Prada (Frankel, 2006). Nous avons choisi de porter notre attention sur le cas spécifique du parcours initiateur de ce projet : celui de Paris, je t'aime.

Le document présentant le parcours a d'abord fait l'objet d'une édition papier de quelques milliers d'exemplaires. Il est aujourd'hui accessible à tous depuis l'Internet en format téléchargeable (Mission cinéma, Parcours cinéma : Paris, je t'aime, www.cinema.paris.fr). Le document bilingue se 
ILLUSTRATION 1 :

Vues de la Place des Fêtes (photos et montage : Michaël Bourgatte).

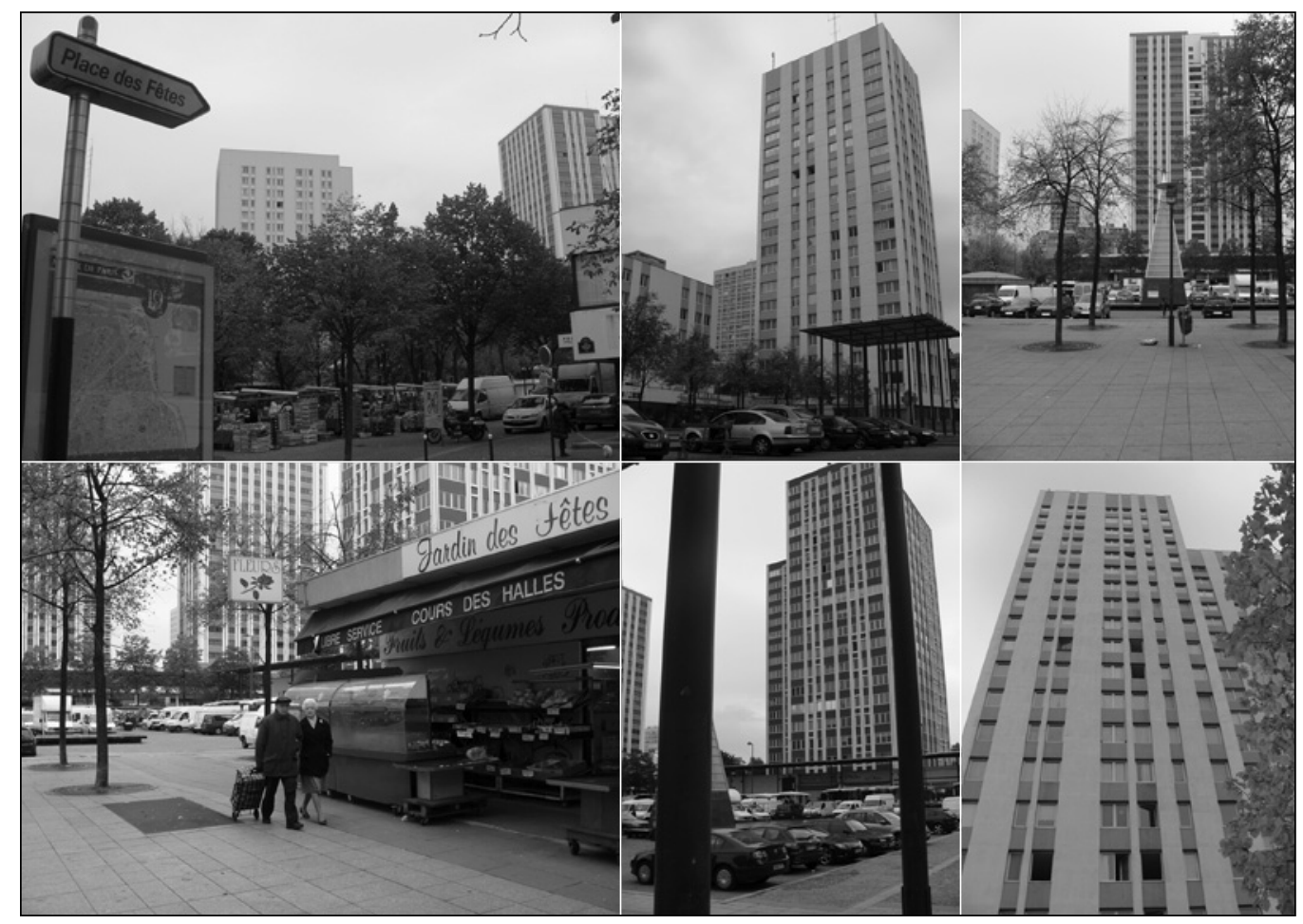

compose de cinq pages. La première page de couverture donne à voir l'affiche du film et les logos institutionnels de la mairie de Paris ainsi que le slogan "Découvrez les lieux de tournage. Discover where the film was shot». La deuxième page du document expose le projet global des parcours cinéma puis présente le film succinctement : «Raconter en cinq minutes l'histoire d'une rencontre amoureuse dans un quartier de Paris, tel est le défi qu'ont accepté de relever, avec Paris, je t'aime, vingt et un réalisateurs venus du monde entier» (mairie de Paris, $2010: 2$ ). La troisième page s'organise autour d'un schéma réduit de Paris segmenté en arrondissements. Sur ce plan sont distribués les points de localisation des lieux de tournage des différents courts métrages composant le film. Six d'entre eux font enfin l'objet d'une attention spécifique en pages 4 et 5 . Il s'agit de la Place des fêtes, du Faubourg Saint-Denis, de Bastille, de la Place des Victoires, du Quais de Seine et du $14^{\mathrm{e}}$ arrondissement (à ce propos, on remarquera que c'est la diversité des types de lieux représentés qui a été privilégiée : il peut s'agir d'une place, d'un quartier, voire d'un arrondissement).

Prenons le cas du premier de ces lieux, la Place des Fêtes, où a été tourné le court métrage d'Olivier Schmitz. Le document présente l'intrigue en un paragraphe : «Hassan a reçu un coup de couteau et agonise sur le terre-plein de la Place des Fêtes. Sophie, une infirmière, arrive à son secours. Il réalise qu'il l'avait aperçue auparavant et que cela aurait pu changer sa vie...» (mairie de Paris, $2010: 4$ ). S'ensuit un second paragraphe qui présente, lui, la Place des Fêtes : «C'était à l'origine un terrain planté de vignes, d'arbres fruitiers et de luzerne. Il fut acquis en 1835 par la municipalité de Belleville pour y organiser les fêtes patronales ou foraines. Aujourd'hui résolument moderne, le terre-plein central a fait l'objet d'un réaménagement par l'architecte Bernard Huet» (mairie de Paris, $2010: 4$ ). Tous les lieux de tournage sont présentés sur ce modèle : un premier paragraphe qui resitue l'intrigue du film et un second paragraphe qui évoque l'histoire du lieu dans lequel le court métrage a été tourné. Ainsi, la présentation de l'intrigue du court métrage et le savoir à caractère institutionnel sur le lieu occupent deux registres de discours distincts qui ne sont aucunement mis en perspective. La seule raison de leur coprésence dans le dispositif tient au référent topographique de leur discours.

Cette Place des Fêtes, si elle est le lieu d'une culture au sens anthropologique, est bien moins volontiers associée à la représentation que l'on peut se faire de la culture comme corpus d'œuvres (Passeron, 1991, r. 2006). Le paysage urbain répond clairement à des attentes liées aux contraintes d'habitat et aux besoins pratiques de la vie quotidienne. En somme, il s'agit davantage d'un espace vécu que d'un espace spectacularisé (voir illustration 1). C'est ce que laisse d'ailleurs entendre la formule quelque peu euphémisante du parcours Paris, je t’aime : «résolument moderne» (mairie de Paris, $2010: 4$ ), lorsqu'il est question de décrire cette place du $19^{\mathrm{e}}$ arrondissement. La description de ce petit dispositif éditorial révèle ainsi la manière dont un lieu de pratiques urbaines ordinaires (la Place des Fêtes) se meut en un lieu de pratiques touristiques extraordinaires (un site culturel qui «vaut le détour»). Cet espace qui, dans d'autres circonstances, pourrait apparaître comme un simple lieu conjonctif se voit requalifié par le dispositif touristique comme un lieu qui exige station et contemplation. Autrement dit, on voit ici qu'un fragment d'espace ordinaire se transforme en chose à voir. 


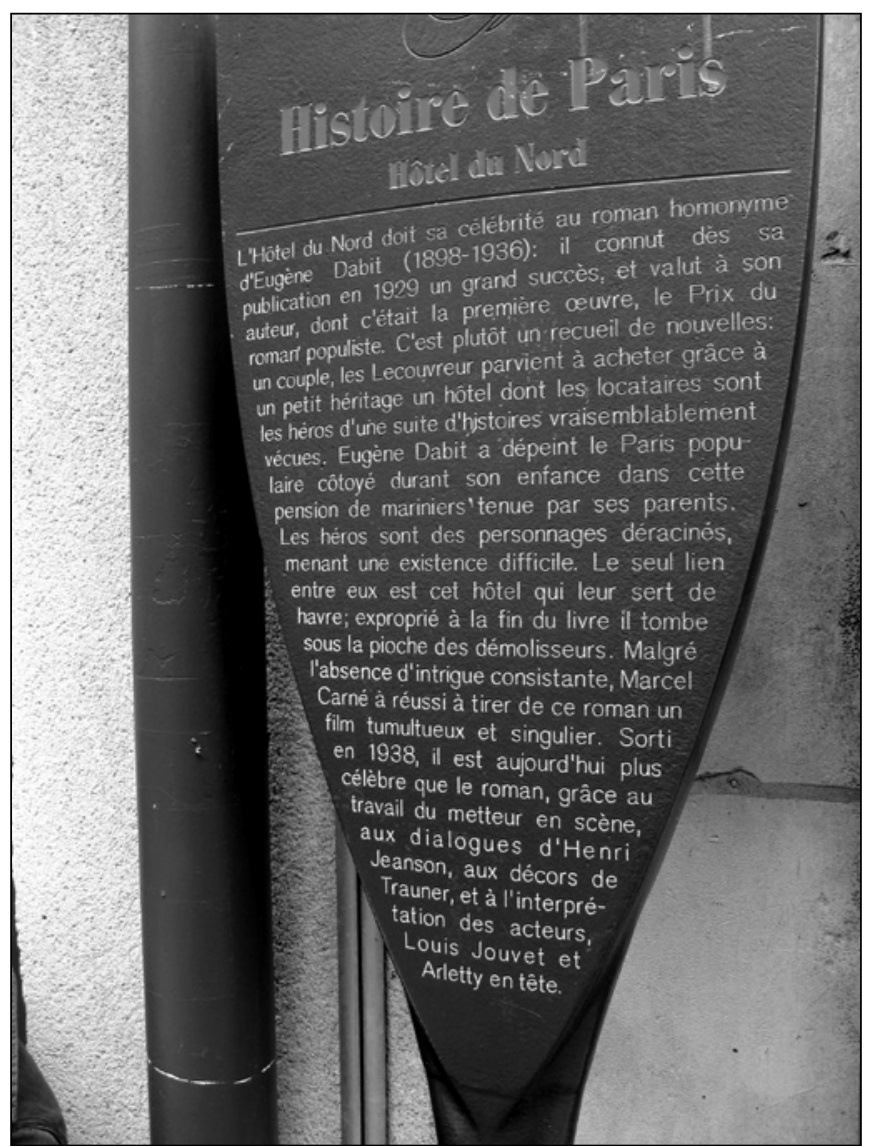

ILLUSTRATION 2 : Un exemple de panneau touristique installé par la mairie de Paris (photo : Michaël Bourgatte).

\section{Le film comme opérateur touristique}

Le film occupe une place fondamentale dans ce processus de requalification du lieu. On notera que le paragraphe de présentation du court métrage apparaît systématiquement avant celui qui traite de l'histoire institutionnelle. Dans le cas que nous avons décrit, celui de la Place des Fêtes, on remarque que le lieu est d'abord resitué dans son contexte filmique avant que soit abordée la question de son contexte historique. Le film s'impose donc comme un «seuil» de l'espace textuel de ces Parcours cinéma, mais aussi comme un «seuil» de l'espace topographique (Genette, 1987). En somme, il apparaît comme le prétexte d'un détour discursif et spatial qui rend le site de la place des Fêtes signifiant d'un point de vue touristique et culturel. Dès lors, c'est parce que le film a été convoqué que ce lieu s'impose comme une chose à voir.

Le film permet d'établir un contact touristique avec le site de la Place des Fêtes. Il se présente ainsi comme un «marqueur» au sens de Dean MacCannell (1976, r. 1999). En effet, un marqueur ne se définit pas seulement comme l'ensemble des informations situées dans l'espace de visite (les panneaux de la mairie de Paris - ou pelles Starck — que l'on retrouve sur le parvis de Notre Dame, dans le Marais ou encore devant l'hôtel du Nord - voir illustration 2). Il enserre tout type d'information qui traite d'un site, qu'elle soit contenue dans un guide de voyage, dans un dépliant de musée ou dans le discours oral d'un professionnel du tourisme. En somme, le marqueur, situé ou non, qualifie, dans un cadre touristique, le rapport que l'on peut entretenir avec certains fragments d'espace. Dire que le film est un marqueur touristique des Parcours cinéma de la ville de Paris, cela revient à définir l'objet filmique comme l'opérateur de la transformation du lieu en site touristique.

Plus largement, il n'est pas rare de voir un film être convoqué à l'intérieur d'un dispositif de médiation touristique pour être assigné à une fonction de marqueur. Au hasard de quelques lectures, on observe que le film When Harry Met Sally, de Rob Reiner (1989), est souvent mobilisé comme opérateur de la transformation d'un simple delicatessen — le Katz's Deli situé dans le Lower East Side new-yorkais - en site touristique, comme le montrent ces deux extraits de guides de voyage : "Vous vous souvenez certainement de la scène du film Quand Harry rencontre Sally quand Meg Ryan feint un orgasme en plein milieu d'un restaurant... Vous y êtes!» (Auzias et Labourdette, 2005 : 203). Ou encore : "C'est là que se déroule la fameuse scène de l'orgasme avec Meg Ryan dans le film Quand Harry rencontre Sally» (Greenfield et Reid, 2005 : 180). Dans les deux cas, par la rhétorique du «c'est là », la référence au film introduit la description du site et lui confère sa valeur touristique.

La stratégie des acteurs et la rhétorique de la trace Cette deuxième partie aborde la question de la mise en cinétourisme des lieux qui ont servi à des tournages. En partant du cas particulier du café des 2 Moulins, cadre privilégié de plusieurs scènes du film Le fabuleux destin d'Amélie Poulain (Jeunet, 2001), nous nous intéresserons, plus précisément, à la stratégie des acteurs (ici, les propriétaires du café) en analysant la façon dont ils mettent en scène le film dans ce lieu.

\section{La mise en scène des objets}

Le café des 2 Moulins est situé dans la rue Lepic du 18 arrondissement de Paris, au cour du célèbre quartier Montmartre. Le réalisateur français Jean-Pierre Jeunet y a tourné plusieurs scènes du film qui l'a rendu célèbre auprès du public international: Le fabuleux destind'Amélie Poulain. Ce bar, qui se trouve à proximité du célèbre cabaret le Moulin Rouge, duquel il tire son nom, est aujourd'hui un lieu de passage obligé pour de nombreux touristes. Une fréquentation répétée de ce lieu nous a permis de nous en rendre compte. On y croise de nombreux individus de toutes nationalités armés d'appareils photos et de guides touristiques. La plupart d'entre eux s'empressent d'ailleurs de photographier en contre-plongée la devanture du café, dès leur arrivée, à la façon dont Jean-Pierre Jeunet l'a mise en scène dans son film (voir illustration 3 ).

Si ce sont les dispositifs de médiation qui ont garanti aux touristes que ce café «valait le détour», les gérants du lieu n'hésitent pas à les conforter dans l'idée qu'ils ne se sont pas trompés : c'est bien le café des 2 Moulins dans lequel travaille Amélie Poulain. En effet, le film est convoqué de diverses façons. Dans la salle, l'affiche du film en grand format est mise en évidence dans un cadre ovale et doré qui rappelle la texture du film. Un nain de jardin trône fièrement à proximité de 
celle-ci, évoquant un épisode rocambolesque du récit : celui du voyage improbable de cette créature de terre cuite qui s'est enfuie du potager du père d'Amélie pour parcourir le monde et se faire photographier aux États-Unis, en Inde et ailleurs. Dans les toilettes, on trouve une série d'objets parmi lesquels : les polaroïds du susmentionné nain de jardin pris aux quatre coins de la planète, la lampe de chevet de Collignon — un des protagonistes de l'histoire - , une autre affiche promotionnelle ou encore des photos de tournage (voir illustration 4).

Ces objets, présents dans les toilettes, sont rassemblés dans une vitrine, à la manière d'un petit mausolée. Ainsi, contrairement à l'affiche et au nain de jardin de la salle principale, tout contact tactile est rendu impossible. Ils se donnent à voir comme des objets singuliers que l'on chercherait à soumettre à un traitement spécifique qui rappelle celui des objets de collection (Pomian, 1987). Par ailleurs, aucun texte ne vient expliciter leur situation de «monstration» (Davallon, 1999). Toutefois, il est possible de dire, à la façon dont ils sont orientés, qu'ils se mettent à disposition du regard des «commissionneurs». Autrement dit, ils sont «mis en exposition» (Davallon, 1999). On s'intéressera à la «fonction-signe» de ces objets $(E c 0,1980)$ et on cherchera à identifier le type de rapport qu'ils construisent avec ce qu'ils représentent en tant que signes.

\section{Les objets comme indices, icones et symboles du film}

En premier lieu, on notera que ces objets ont des statuts divers. Ils n'établissent pas le même type de rapports avec le référent filmique. Certains semblent avoir été prélevés de l'univers narratif du film quand d'autres ont été produits en vue de sa promotion et de sa circulation médiatique. On tentera d'affiner cette observation en mobilisant l'approche tripartite du signe chez Peirce, non pas pour dégager une logique d'interprétation des signes, mais pour mettre en évidence trois stratégies différentes de l'élaboration des signes. Rappelons-le pour mémoire, cette approche repose sur une distinction entre l'indice, l'icone et le symbole (Peirce et Deledalle, 1978). L'indice est un signe qui entretient un rapport contigu avec la chose qu'il désigne. Au départ, il lui était attaché. Institué en tant qu'indice, il s'en voit arraché pour mieux la représenter. L'icone est un artefact qui s'ajoute au monde. Elle établit un rapport analogique avec l'objet qu'elle représente. Enfin le symbole rompt toute analogie et toute contiguïté avec l'objet qu'il signifie. Il est ainsi un signe proprement arbitraire.

Dans la vitrine des toilettes du café des 2 Moulins, les polaroïds du nain de jardin et la lampe de chevet de Collignon construisent un rapport indiciel au film. Ils semblent en avoir été arrachés pour se voir exposés. On pourra rétorquer qu'ils sont possiblement faux. Quand bien même ce serait le cas, ces copies seraient à ce point conformes aux originaux qu'elles n'en cesseraient pas moins de se donner comme appartenant à l'univers du film. Les nains de jardin définissent, quant à eux, un rapport iconique au film. Leur morphologie n'est pas celle du héros voyageur farceur qu'on y rencontre. Aucun d'entre eux n'a donc pu être arraché au film. Toutefois, la dynamique de coprésence de plusieurs de ces petits êtres permet d'instituer une relation de ressemblance ou d'analogie avec le nain

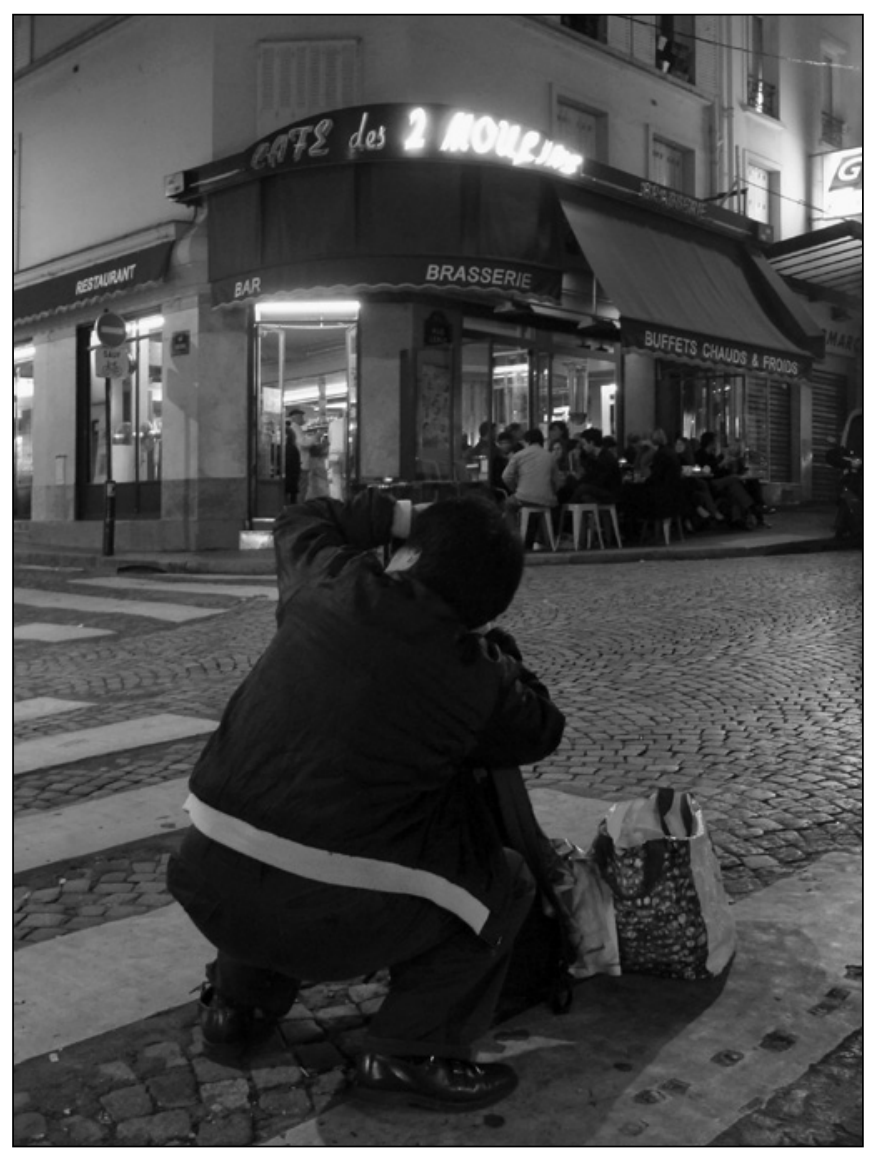

ILLUSTRATION 3 : Un touriste en train de photographier la devanture du café des 2 Moulins (photo : Michaël Bourgatte).

de jardin du Fabuleux destin d'Amélie Poulain. Enfin, l'affiche instaure un rapport symbolique avec le film. Elle l'évoque de façon conventionnelle en tant qu'elle est un opérateur de promotion (inscriptions sur l'affiche : titre, noms du réalisateur et des acteurs, logos, etc.).

Ces catégories d'analyse s'appliquent plus largement aux objets exploités par les acteurs touristiques pour convoquer les films dans le cadre d'une activité ciné-touristique. Ainsi, le site Internet du restaurant l'hôtel du Nord affiche clairement sa filiation avec le film de Marcel Carné, Hôtel du Nord (1938). Sur la page d'accueil, on peut entendre la voix d'Arletty déclamer une des répliques du film parmi les plus célèbres de l'histoire du cinéma : "Atmosphère! Atmosphère! Est-ce que j’ai une gueule d'atmosphère?». On a affaire, ici, à un cas original de signe indiciaire proprement sonore. En Nouvelle-Zélande, à proximité de Matamata, une ferme s'est vue transformée en Hobbiton pour les besoins du tournage de la trilogie The Lord of the Rings (Jackson, 2001, 2002 et 2003). Seule une petite partie des décors est restée en place. Cependant, des panneaux disposés sur le site remplissent une fonction iconique en donnant à voir le village des hobbits tel qu'il apparaît dans le film. Enfin, le Twede's cafe (le Double R Diner de Twin Peaks), en nommant l'un des hamburgers de sa carte du nom de cette ville imaginaire, fait un clin d'œil au film (Lynch, 1991) et à 


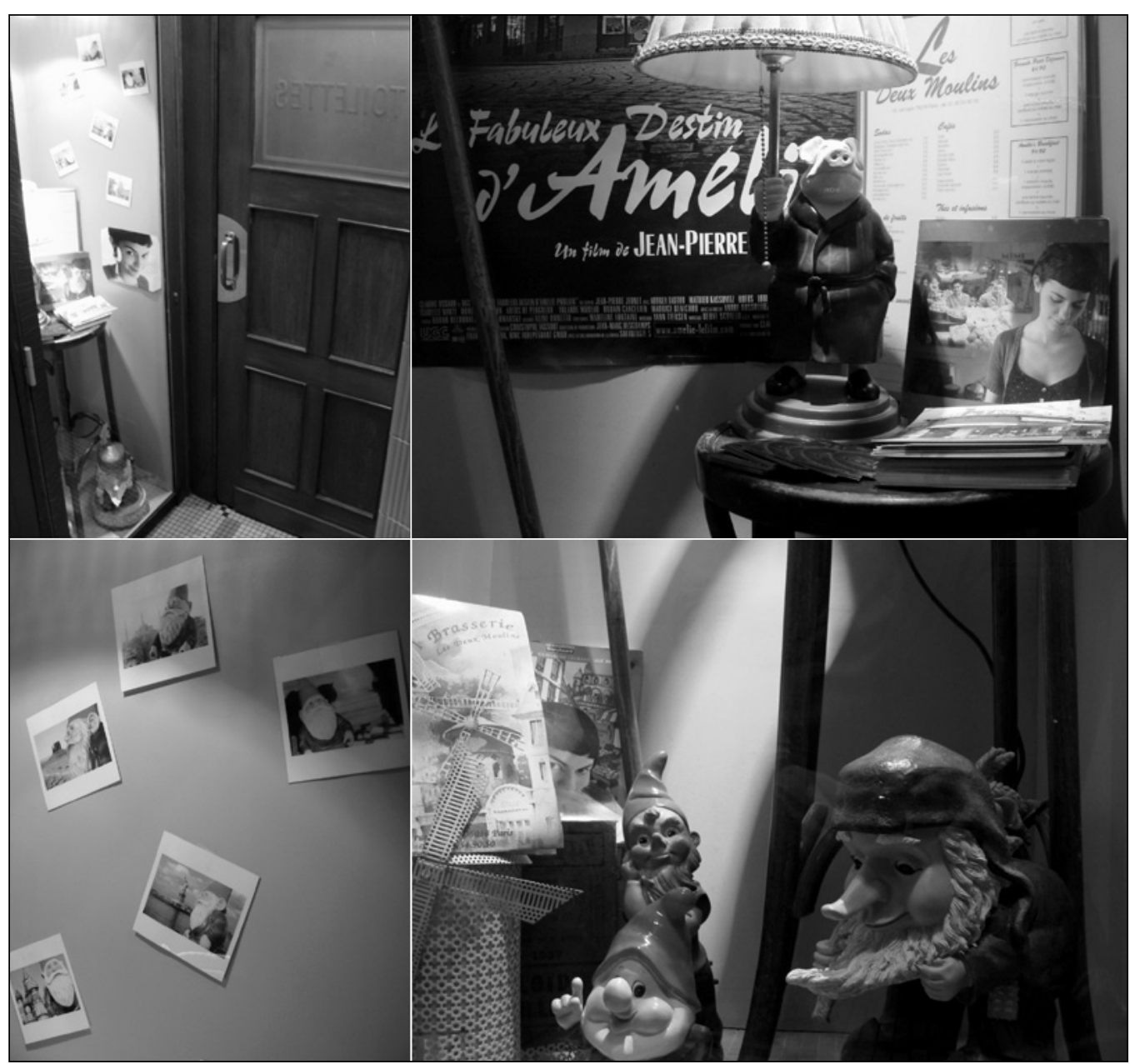

\section{ILLUSTRATION 4 :}

Vues de la vitrine du café des 2 Moulins (photos et montage : Michaël Bourgatte). la série (Lynch et Frost, 1990) qui l'ont mis en scène. Il choisit ainsi de construire avec ces œuvres de fiction un rapport de l'ordre du symbolique.

\section{La question de la trace située}

On voit que ces lieux, et à plus forte raison le café des 2 Moulins du Fabuleux destin d'Amélie Poulain, ont choisi de se mettre en scène en rassemblant divers types d'objets qui font sens en tant que signes. En les livrant à la vue et à l'ouïe du visiteur, ils cherchent à mettre en évidence la relation qu'ils entretiennent avec le film. Ainsi, ils montrent ces objets pour rendre présent le film en donnant à voir une part de ce qui lui subsiste. On se demandera cependant si ces objets conserveraient le même statut dans un espace d'exposition autre n'entretenant aucune relation avec les espaces mis en scène dans le film. Pour le dire autrement, la lampe de chevet de Collignon conserve-t-elle le même statut qu'elle soit exposée dans la vitrine du café des 2 Moulins ou à la Cinémathèque Française?

Pour répondre à cette question, on mobilisera la notion de trace. Alexandre Serres note que «la trace se caractérise par son génitif intrinsèque, si l'on peut dire, i. e. son caractère d'appartenance, au sens où la trace est toujours trace de quelque chose» (Serres, 2002). Par ailleurs, comme le fait remarquer
Paul Ricœur, la trace est la présence ou «la représentation présente d'une chose absente» (2000 : 746) ; en somme, elle continue à faire exister ce qui a été. Les nains de jardin, les photos ou la lampe de Collignon, lorsqu'ils sont exposés dans les toilettes du café des 2 Moulins, se livrent comme des traces du film. Dans ce cas, les objets sont situés dans leur univers narratif. S'ils se voyaient, par exemple, exposés à la Cinémathèque Française, ils se verraient incontestablement arrachés à cet univers. Autrement dit, dans le premier cas, il s'agit de ce que nous appellerons des traces situées; par effet d'opposition, nous appellerons les secondes des traces non situées. La spécificité des traces situées est qu'elles renvoient au film à la fois comme univers narratif (ce lieu dans lequel travaille Amélie), et au film comme produit culturel réalisé dans le cadre d'une pratique professionnelle (le café des 2 Moulins transformé en décor le temps d'un tournage).

La trace située nous ramène à la rhétorique du "c'est là », évoquée précédemment à propos du film When Harry Met Sally, qui cherche à confondre espace de pratiques ordinaires et espaces narrativisés extraordinaires. Ainsi, qu'elle soit indicielle, iconique ou symbolique, la trace située participe de la requalification des lieux. Le petit Hôtel du Nord de Marcel Carné devient "l'hôtel du Nord», un passage obligé 
pour les touristes cinéphiles alors que le film n'y a même pas été tourné puisque l'essentiel de la production cinématographique, jusque dans les années 1950, faisait l'objet de tournages en studio. L'île grecque de Skopelos devient, quant à elle, «l'île de Mamma Mia!» (Lloyd, 2008) alors qu'elle n’a pas été la seule à servir de décor au film, celui-ci ayant également été tourné sur l'île de Skiathos et à Damouchari, sur le littoral grec. Ainsi, la mise en scène de la trace située a pour objectif d'engendrer une mise en tourisme des lieux. Celle-ci est le fait d'une stratégie d'acteurs qui cherchent à faire exister des lieux appartenant à des univers narratifs cinématographiques dans un réseau de sites touristiques.

\section{La boucle allographique du ciné-tourisme}

Dans un premier temps, nous avons vu que les dispositifs de médiation touristique participent à la mise en ciné-tourisme de lieux de tournage. Nous avons, dans un second temps, montré comment ces lieux exploitent le film à des fins touristiques. La question que l'on se posera dans ce dernier point est la suivante : pourquoi tous les lieux de tournage ne sont pas mis en tourisme? Autrement dit, pourquoi certains lieux plus que d'autres connaissent une carrière touristique?

\section{Le cinéma et les destinations stéréotypées}

Comme l'ont montré Siegfried Kracauer (1947, r. 1973) et, avant lui, Erwin Panofsky (1936, r. 1995), les films portent en eux quelque chose des sociétés qui les ont produits. Ils dévoilent ainsi l'état du monde au moment où ils le dépeignent. S'ils mettent en scène les aspirations des acteurs sociaux, ils révèlent également les lieux communs entendus, ici, au sens de topoï, c'est-à-dire un ensemble de représentations que les acteurs sociaux ont en partage et sur lesquelles ils se mettent d'accord pour fonder un système de références et de valeurs communes.

Dans le cas de Mamma Mia!, les lieux communs sont multiples. L'image de la Grèce qui est construite dans ce film est faite de vieilles femmes vêtues de noir et portant des fagots de sarments sur leur dos, d'insulaires à béret, ridés et tannés par le soleil, de petites embarcations de bois voguant sur les eaux azures et transportant de concert hommes et volailles. En somme, on y retrouve tout ce qui peuple les cartes postales que le touriste peut se procurer dans ces kiosques grecs que sont les periptera. On reconnaîtra, dans toutes ces figures, ce que l'on appelle communément des «stéréotypes». Si l'on en croit Ruth Amossy, ceux-ci agissent comme des instruments de repérage socioculturel. Admis et largement diffusés, ils apparaissent comme «l'équivalent de l'objet standardisé dans le domaine culturel» (Amossy, $1991: 21$ ). La veuve, le vieux loup de mer ou la barque sont autant d'images préfabriquées de la Grèce qui préexistent au film mais que ce dernier remet en circulation.

Pour Ruth Amossy, ces images préfabriquées ne sont pas condamnables. L'auteure explique qu'il faut distinguer le stéréotype de la "conscience du stéréotype»:

Le premier désigne un phénomène de portée universelle; on imagine mal une collectivité qui ne possède en commun un minimum d'images, d'opinions et de croyances. La seconde est au contraire propre aux temps modernes : il faut attendre le XIX ${ }^{\mathrm{e}}$ siècle pour que la banalité d'expression et de pensée soit partout traquée, et le $\mathrm{XX}^{\mathrm{e}}$ pour que l'image collective d'une catégorie sociale donnée soit perçue comme un stéréotype, c'està-dire une schématisation outrée (1991: 10).

On s'accordera donc avec cette proposition pour sortir du système de jugement de valeur qui accompagne la conscience du stéréotype afin de ne prendre en compte que la valeur représentationnelle de ces figures que le film convoque.

\section{La fabrique de l'authenticité}

La raison d'être de ces stéréotypes repose sur un désir de véhiculer de "l'authenticité». Nous n'entendrons pas cette dernière comme le résultat d'une procédure d'authentification conduite par des experts qui ont pour mission d'identifier la valeur des objets afin d'en préserver l'intégrité : action des conservateurs de musées, des chercheurs de l'Inventaire du patrimoine culturel ou autres (Heinich, 2009; Morisset, 2009). Cette notion renverra aux représentations communes, à ce que Christian Bessy et Francis Chateauraynaud appellent «une croyance collective» (1995:229). L'authenticité sera donc comprise comme le résultat d'une mise en accord des acteurs sociaux (institutions, professionnels du tourisme et touristes) autour d'un régime d'existence des objets, des lieux et des êtres qui auraient réussi à préserver quelque chose d'eux-mêmes dans le temps (MacCannell, 1976, r. 1999).

L'authenticité, ainsi définie, nous éclaire sur la question de la carrière ciné-touristique de lieux mis en scène dans des films. Revenons sur le cas de Mamma Mia! et de la Grèce. Bien avant la sortie du film, ce pays était déjà construit comme une destination touristique faite de vestiges et de ruines. Elle est cet "univers finissant», "rescapé du passé», une destination "dominée par l'esthétique du débris» et peuplée de «fossile[s] vivant[s]» (Urbain, $1991: 216 s q$.). En mobilisant les stéréotypes évoqués ci-dessus, le film ne fait que renforcer cette image «touristiquement authentique » de la Grèce et plus particulièrement de ses territoires insulaires du bout du monde. Les acteurs locaux, plutôt que d'aller contre, bien au contraire, s'emparent de cette image pour la confirmer à leur tour. Il en va de même pour Le fabuleux destin d'Amélie Poulain qui joue la carte de l'«authentique parisianité» et donne ainsi l'occasion au café des 2 Moulins de se manifester dans le monde touristique comme un signe sursignifiant de cette "parisianité». Quant au film When Harry Met Sally, il exploite le filon d'une américanité débridée, d'une vie new-yorkaise volubile.

Ces films ne mettent pas simplement en scène des lieux ordinaires, mais des lieux qui existent déjà dans les représentations comme des sites ou des destinations touristiques. Ces derniers, pour se légitimer en tant que tels, n'hésitent pas à multiplier les références au film. Autrement dit, si certains lieux exploités par le cinéma ont une carrière cinétouristique plus importante que d'autres, c'est que, d'une part, leur mise en scène renforce les «horizons d'attentes » (Jauss, 1972, r. 1990) des touristes et, d'autre part, que ces lieux choisissent de concevoir une stratégie touristique qui correspond à des «espaces d'expériences» attendues 
(Koselleck, 1990). Ainsi, les films qui convoquent diverses figures stéréotypées, et les acteurs qui en tirent parti, renforcent l'identité touristique des lieux, c'est-à-dire leur existence en tant que destinations.

\section{L'agir allographique}

Cette pratique de l'écriture de la destination dans le film et le maintien de traces du film dans les lieux ciné-touristiques définissent ce que nous appellerons une «allographie touristique». On sortira du cadre esthétique établi par Gérard Genette (1987) pour penser l'allographie comme une pratique d'inscription plus générale qui ne concerne pas uniquement les objets pris dans leur dimension artistique. L'allographie sera entendue au sens étymologique de pratique scripturaire de l'autre, qu'elle engage ou non des textes littéraires. On dira ainsi que Mamma Mia! et les acteurs touristiques de Skopelos établissent ensemble une allographie touristique autour de la figure de Kalokairi, le nom de l'île dans le film, signifiant «été» en grec moderne.

Par ailleurs, l'allographie touristique ne doit pas seulement être saisie comme une écriture réciproque du lieu dans le film et du film dans le lieu. Elle est aussi à l'œuvre lorsque des dispositifs de médiation mettent en relation le film et le site touristique. Quand les guides de voyage de New York précisent que le Katz's Deli est un lieu qui mérite une attention particulière parce qu'il a servi de décor au film When Harry Met Sally, ils mettent en relation le film et le lieu, et procèdent à une écriture touristique proprement allographique. Ce qui apparaît comme une «surenchère communicationnelle» potentialise les effets ciné-touristiques de l'écriture allographique.

Les dispositifs de médiation touristique comme les guides de voyage ou les plaquettes Parcours cinéma de la mairie de Paris ont donc un rôle fondamental à tenir dans ce que nous appelons "la boucle allographique». En effet, ces tiers dispositifs ont le pouvoir de délester les destinations de leur charge stéréotypante en déplaçant les figures de l'authenticité au moyen de nouveaux marqueurs filmiques. Lorsque la Place des Fêtes du $19^{\mathrm{e}}$ arrondissement est travaillée comme un site touristique par la mairie de Paris, cette dernière opère une rupture vis-à-vis de l'identité touristique d'un Paris à la Amélie Poulain pour attirer le regard sur des sites qui, en dehors d'une médiation filmique, ne feraient pas tourisme. En somme, les acteurs de la médiation touristique ont le pouvoir de reconfigurer des identités à condition, bien sûr, qu'ils fabriquent de nouvelles traces afin de rendre possible la surenchère communicationnelle évoquée précédemment.

\section{Conclusion}

En 2004, les éditions Parigramme ont publié un petit guide touristique de Paris à l'usage des cinéphiles (Renouard, 2004, r. 2006). Intitulé Paris cinéphile, cet ouvrage propose aux ciné-touristes de visiter des salles dites "mythiques» de la capitale française comme le Balzac et l'Accattone. Il suggère encore d'aller visiter des lieux d'exposition ou de conservation liés à l'activité cinématographique, comme le Musée du Cinéma ou la structure d'expositions itinérantes joliment nommée Le cinéma s'expose. On voit donc que ce guide a composé un territoire ciné-touristique à partir de lieux que l'on qualifiera d'institutionnels et qu'il a laissé de côté la part des lieux ordinaires qui pourtant configurent tout autant ce territoire à l'instar du café des 2 moulins ou de l'hôtel du Nord.

Le présent article a tenté de les mettre à l'honneur pour deux raisons. La première est que l'analyse de ces lieux représente un défi, car ils ne disent rien, à priori, du cinéma : ce sont des lieux de la quotidienneté que l'on peut fréquenter en dehors de toute activité touristique. La seconde est que ces lieux participent pleinement du circuit culturel, économique, pratique et symbolique du ciné-tourisme. Nous avons vu que de nombreux touristes se rendent volontiers dans le café d'Amélie Poulain pour y vivre quelque chose du Paris de Jean-Pierre Jeunet. Les guides de voyage, quant à eux, n'hésitent pas à inclure, dans la liste des choses à voir, des lieux tels que le Katz's Deli du film When Harry Met Sally comme si un événement aussi insolite pouvait s'y produire. Pourtant, ces deux lieux n'ont rien fait d'autre que d'abriter un tournage. Ils n'ont été, en somme, que les témoins d'une activité humaine passée. Pourquoi cet intérêt, dès lors, pour ce type de lieux? Pourquoi les dispositifs de médiation et la pratique des touristes cherchent ensemble à en faire des sites ou des destinations ciné-touristiques? Comment ceux-ci le deviennent-ils? C'est à ces questions que nous avons tenté de répondre.

Il est apparu que les acteurs du ciné-tourisme convoquent deux grandes catégories d'objets : les marqueurs et les traces. Les premiers, exploités par les médiateurs touristiques, se définissent comme des références aux univers narratifs cinématographiques. Ils sont des opérateurs de transformation de simples fragments d'espaces ordinaires en lieux testimoniaux extraordinaires. Ils sont, autrement dit, des instruments de la requalification de lieux ou de territoires en sites ou en destinations proprement touristiques. Les secondes, lorsqu'elles sont situées - c'est-à-dire mises en scène par les lieux eux-mêmes - sont des instruments de confirmation de la valeur ciné-touristique de ces lieux.

D'une manière générale, marqueurs et traces permettent de mettre en évidence le fait que le ciné-tourisme se définit comme le résultat d'une chaîne de coopération entre les acteurs du ciné-tourisme : c'est ce que nous avons appelé la stratégie allographique. Les propriétaires ou gérants des lieux doivent savoir tirer profit de l'image que le film construit de leurs espaces. Ils doivent ainsi savoir organiser un système de traces. Les médiateurs touristiques doivent, de leur côté, poser le film comme un opérateur de requalification des lieux ordinaires en lieux ciné-touristiques en instituant celui-ci comme marqueur.

Cette économie du discours allographique, nous l'avons $\mathrm{vu}$, repose sur une somme de préconstruits sociaux et culturels, à savoir les stéréotypes. L'allographie ciné-touristique peut donc prendre deux directions : elle peut travailler au renforcement des stéréotypes en proposant de construire des lieux ciné-touristiques qui correspondent aux horizons d'attente des voyageurs; elle peut également choisir de les rompre. Dans le premier cas, il existe un risque de réduction, 
de fixation, voire de folklorisation des cultures (Barthes, 1957). Dans le second cas, le ciné-tourisme devient un enjeu de la redéfinition et de la renégociation de l'identité des sites et des destinations. Ainsi, des institutions comme la mairie de Paris peuvent choisir de retravailler l'identité de certains lieux en mobilisant les ressources du ciné-tourisme. C'est ce que nous avons montré avec l'étude du dispositif de médiation présentant la Place des Fêtes. Pour ce faire, ces institutions doivent cependant savoir se saisir de productions cinématographiques singulières et hors-normes, au sens où elles déconstruisent la dimension stéréotypante de ces lieux afin d'en produire une nouvelle.

\section{Références}

ADAM, Jean-Michel (2001) «Types de textes ou genre de discours? Comment classer les textes qui disent de et comment faire?», Langages, vol. 35, nº 141, p. 10-27.

AMOSSY, Ruth (1991) Les Idées reçues. Sémiologie du stéréotype, Paris : Nathan. 215 p.

AUZIAS, Dominique et Jean-Paul LABOURDETTE (2005) Petit Futé : NewYork 2005-2006, Paris : Nouvelles Éditions de l’Université. 448 p.

BARTHES Roland (1957) «Le Guide bleu», DANS Mythologies, p. 121-125. Paris : Le Seuil.

BESSY, Christian et Francis CHATEAURAYNAUD (1995) Experts et faussaires - Pour une sociologie de la perception, Paris : Métailié. $364 \mathrm{p}$.

CASAZZA, Christophe (2003) Ciné Paris - 20 balades sur des lieux de tournages mythiques, Paris : Presses de la Cité. 167 p.

CERTEAU, Michel de; Luce GIARD et Pierre MAYOL (1980, r. 1990) L'Invention du quotidien - 1. Arts de faire, Paris : Gallimard. 349 p.

COHEN, Martin (2006) No holidays - 80 places you don't want to visit..., New York : Desinformation Company. 191 p.

DAVALLON, Jean (1999) L'exposition à l'œuvre. Stratégies de communication et médiation symbolique, Paris : L'Harmattan. 378 p.

DAVALLON, Jean et Yves JEANNERET (2006) «La posture épistémologique, un geste pratique», DANS CHEVALIER, Yves (dir.), Questionner les pratiques d'information et de communication : agir professionnel et agir social. Actes du XVI ${ }^{e}$ Congrès de la SFSIC (Bordeaux, 10 au 12 mai 2006). Paris : Jouve/SFSIC, p. 203-210.

ECO, Umberto (1980) «Peirce et la sémantique contemporaine», Langages, vol. 14, n 58 , p. 75-91.

GENETTE, Gérard (1987) Seuils, Paris : Éditions du Seuil. 426 p.

GREENFIELD, Beth et Robert REID (2005) Lonely Planet New-York, Paris : Lonely Planet. 392 p.

GRITTI, Jules (1967) «Les contenus culturels du Guide bleu : monuments et sites “à voir" ", Communications, vol. 10, n 10, p. 51-64.
HEINICH, Nathalie (2009) La fabrique du patrimoine. De la cathédrale à la petite cuillère, Paris : Editions de la Maison des Sciences de l'Homme. $286 \mathrm{p}$.

HYMAN, Miles et Vincent REA (2010) New York, itinéraires, Bruxelles : Casterman. $159 \mathrm{p}$.

JAUSS, Hans Robert (1972, r. 1990) Pour une esthétique de la réception, Paris : Gallimard. 333 p.

KOSELLECK, Reinhart (1990) Le futur passé: contribution à la sémantique des temps historiques, Paris : EHESS. $334 \mathrm{p}$.

KRACAUER, Siegfried (1947, r. 1973) De Caligari à Hitler : une histoire psychologique du cinéma allemand, Lausanne : L'âge d'homme. 409 p.

MACCANNELL, Dean (1976, r. 1999) The Tourist - A New Theory of the Leisure Class, Los Angeles : University of California Press. $231 \mathrm{p}$.

MAIRIE DE PARIS (2010) «Parcours cinéma dans Paris no 1 : Paris, je t’aime», Mission Paris, format pdf : <http://www.paris.fr/portail/loisirs/ Portal.lut?page_id=8542>, consulté le 15 octobre 2010.

MORISSET, Lucie (2009) Des régimes d'authenticité — Essai sur la mémoire patrimoniale, Rennes : Presses Universitaires de Rennes. $131 \mathrm{p}$.

PANOFSKY, Erwin (1936, r. 1995) "Style and Medium in the Motion Pictures”, DANS LAVIN, Irving, Three Essays on Style, p. 93-125. Cambridge : Mass MIT Press.

PASSERON, Jean-Claude (1991, r. 2006) Le Raisonnement sociologique Un espace non-poppérien du raisonnement naturel en philosophie, Paris : Albin Michel. 666 p.

PEETERS, Benoit et François SCHUITEN (2002) Le guide des Cités, Paris : Casterman. $192 \mathrm{p}$.

PEIRCE, Charles Sanders et Gérard DELEDALLE (1978) Écrits sur le signe, Paris : Le Seuil. 263 p.

POMIAN, Krzysztof (1987) Collectionneurs, amateurs et curieux. Paris, Venise : XVI ${ }^{\text {ème }}$ - XVIII ìme siècle, Paris : Gallimard. $367 \mathrm{p}$.

RENOUARD, Gilles (2004, r. 2006) Paris cinéphile, Paris : Parigramme. 111 p.

RICHARD, Nicolas (2006) La Molvanie — Le pays que s'il existait pas, faudrait l'inventer, Paris : Flammarion. $176 \mathrm{p}$.

RICCEUR, Paul (2000) «L'écriture de l'histoire et la représentation du passé», Annales. Histoire, Sciences Sociales, vol. 55, nº 4, p. 731-747.

SERRES, A. (2002) Quelle(s) problématique(s) de la trace? : communication prononcée lors du séminaire du CERCOR Traces et corpus dans les recherches en Sciences de l'Information et de la Communication (13 décembre). Disponible en ligne sur @rchiveSIC : <http://archivesic.ccsd.cnrs.fr/ sic_00001397.html>.

URBAIN, Jean-Didier (1991) L'Idiot du voyage - Histoires de touristes, Paris : Payot et Rivages. 353 p. 